\title{
Starshade Rendezvous: exoplanet orbit constraints from multi-epoch direct imaging
}

\author{
Andrew Romero-Wolf, ${ }^{\text {a,* }}$ Geoffrey Bryden $\odot,{ }^{a}$ Greg Agnes, ${ }^{\mathrm{a}}$ \\ Jonathan W. Arenberg $\odot,{ }^{b}$ Samuel Case Bradford, ${ }^{a}$ Simone D’Amico $\odot$, \\ John Debes $\odot,{ }^{\mathrm{d}}$ Matt Greenhouse $\odot,{ }^{\mathrm{e}}$ Renyu Hu$\odot,{ }^{\mathrm{a}}$ Steve Matousek, ${ }^{\mathrm{a}}$ \\ Jason Rhodes, ${ }^{a}$ and John Ziemer ${ }^{\mathrm{a}}$ \\ a Jet Propulsion Laboratory, California Institute of Technology, Pasadena, \\ California, United States \\ ${ }^{b}$ Northrop Grumman Aerospace Systems, Redondo Beach, California, United States \\ ${ }^{\mathrm{c}}$ Stanford University, Stanford, California, United States \\ ${ }^{\mathrm{d}}$ Space Telescope Science Institute, Baltimore, Maryland, United States \\ ${ }^{e}$ NASA Goddard Space Flight Center, Greenbelt, Maryland, United States
}

\begin{abstract}
The addition of an external starshade to the Nancy Grace Roman Space Telescope will enable the direct imaging of Earth-radius planets orbiting at $\sim 1$ AU. Classification of any detected planets as Earth-like requires both spectroscopy to characterize their atmospheres and multi-epoch imaging to trace their orbits. We consider here the ability of the Starshade Rendezvous Probe to constrain the orbits of directly imaged Earth-like planets. The target list for this proposed mission consists of the 16 nearby stars best suited for direct imaging, around which $\sim 10$ to 15 planets are expected to be discovered. Of these planets, $\sim 1$ to 2 will be Earth-like in mass and temperature. The field of regard for the starshade mission is constrained by solar exclusion angles, resulting in four observing windows during a two-year mission. We find that for Earth-like planets that are detected at least three times during the four viewing opportunities, their semi-major axes are measured with a median precision of 7 mas, or a median fractional precision of 3\%. Habitable-zone planets can be correctly identified as such $96.7 \%$ of the time, with a false positive rate of $2.8 \%$. If a more conservative criteria are used for habitable-zone classification (95\% probability), the false positive rate drops close to zero, but with only $81 \%$ of the truly Earth-like planets correctly classified as residing in the habitable zone. (C) The Authors. Published by SPIE under a Creative Commons Attribution 4.0 Unported License. Distribution or reproduction of this work in whole or in part requires full attribution of the original publication, including its DOI. [DOI: 10.1117/1.JATIS.7.2.021219]
\end{abstract}

Keywords: planets; space optics; imaging.

Paper 21009SS received Jan. 25, 2021; accepted for publication May 20, 2021; published online Jun. 4, 2021.

\section{Introduction}

The Starshade Rendezvous probe (SRP) mission concept proposes adding a Starshade to the Nancy Grace Roman Space Telescope enabling the detection of habitable zone exoplanets and characterization of their atmospheres. ${ }^{1}$ The detailed technical basis for the SRP study report ${ }^{1}$ was presented in Ref. 2 along with the simulations used to estimate sensitivity of the observatory. The corresponding software is publicly available for reproduction of the results below and for comparison with similar simulations (https://github.com/afromero/Starshade_Rendezvous_ Probe_sims). The main result of these studies is that SRP is capable of discovering Earth-size planets in the habitable zones of nearby stars using the relatively moderate aperture of the Roman space telescope ${ }^{2}$ along with the Coronagraph Instrument.

While the SRP science objectives include quantifying the amount of habitable zone dust around nearby stars and measuring the metallicity of known gas giant planets, the primary

*Address all correspondence to Andrew Romero-Wolf, Andrew. Romero-Wolf@jpl.nasa.gov 
driver is the detection and characterization of Earth-like planets. The overall strategy, described in more detail in Ref. 2, involves three main steps: (1) initial detection via direct imaging, (2) habitable zone determination via orbit tracing, and (3) atmosphere characterization via spectroscopy. The integration times necessary to image and to take spectra of Earth-like planets (steps 1 and 3) were taken into account with a model of the observatory. However, step 2 is more complicated since the observatory field of regard is constrained by solar exclusion angles, typically limiting the target observing windows to two $\sim 30$ day blocks per year-a total of four observing opportunities during the assumed 2-year mission lifetime. Depending on the orientation and phase of a planet's orbit, it may or may not be visible during each of these four observing windows. In Ref. 2, we assumed that detecting the planet during at least three of the four epochs would be sufficient to determine if a planet lies within its parent star's habitable zone. In this paper, we consider this step in more detail, performing multi-epoch orbit fitting for the target list and expected signal-to-noise ratio (SNR) given by the observatory model used in Ref. 2.

Measurement of planetary orbits has been previously modeled for several types of observations - radial velocity, astrometric wobble, and coronagraphic direct imaging. Examples include: (1) combining radial velocity measurements with direct imaging upper limits to improve the orbital fit for the planet eps Eri $b,^{3}$ (2) quantifying the robustness of planetary orbit determination via the stellar astrometric signal, concentrating on the difficulty posed by multi-planet systems, ${ }^{4}$ and (3) considering simultaneous stellar astrometry and direct imaging, finding that Earth-like planets can be characterized in just a few observations. ${ }^{5}$ Among the studies that, such as this paper, concentrate on direct imaging, Ref. 6 performed a detailed analysis of orbital constraints based on the small fraction of an orbit that is traced by known long-period direct-imaged planets. For theoretical cases where the observations span at least half an orbital period, Ref. 7 finds that three equally spaced observations with SNR $\geq 10$ can measure the semi-major axis and eccentricity to $10 \%$. Reference 8 also considered direct imaging of shorter period planets, finding that a habitable zone planet's semi-major axis can be measured to within 5\% if it is observed with precision 3.5 mas over three epochs each spaced by at least 90 days apart.

Here, we consider the results obtainable by a specific mission concept-the SRP. Unlike previous work, this includes: (1) a realistic SNR calculation as a function of stellar illumination, rather than an assumed astrometric precision, (2) a starshade-specific inner working angle (IWA) that obscures planet images close to the star, and (3) observing windows based on known target sky positions and observatory pointing constraints, not just arbitrarily spaced observations. Furthermore, we focus here not on general orbit fitting results, but on a specific science question - whether or not we can determine if a planet lies in its parent star's habitable zone.

In the following, we first summarize the integration time-based observatory model from Ref. 2 used to calculate the SNR for each planet image Sec. 2. For many sets of simulated observations, we apply a parametric SNR-based estimate of astrometric precision to then extract orbital elements for each injected planets Sec. 3. We give the resulting precision for the orbital fits in Sec. 4 and summarize in Sec. 6.

\section{Observing Model}

We briefly summarize the models used for planet properties, orbit propagation, and the observatory. A detailed presentation of these models can be found in Ref. 2.

\subsection{Targets}

Planet sizes and orbital periods are drawn randomly from these defined ranges for Earth-like planets, based on the distribution defined by 13th NASA Exoplanet Program's Study Analysis Group (SAG-13) ${ }^{9}$ and modified by the HabEx mission concept ${ }^{10}$ to include the dependence of the orbital semi-major axis on the lower limit of planet radii. The orbital period $P$ defines the orbital radius $a_{p}$ by way of the stellar mass $M_{\star}$ using Kepler's third law. For sampling of Earth-like exoplanets, the orbits are assumed to be circular, consistent with most previous studies, e.g., Ref. 11. However, when fitting, we allow eccentricity to be a free parameter (see Sec. 3). 
Table 1 Planet and dust parameters.

\begin{tabular}{|c|c|}
\hline Parameter & Value [or(range)] \\
\hline Earth-like planet geometric albedo ${ }^{a}$ & 0.2 \\
\hline Earth-like planet radius & {$\left[0.8 a_{\mathrm{p}}^{-1 / 2} L_{\star}^{1 / 4}-1.4\right] r_{\oplus}$} \\
\hline Habitable zone & {$[0.95-1.67] \sqrt{L_{\star}} \mathrm{AU}$} \\
\hline Zodiacal dust brightness ${ }^{\mathrm{b}}$ & $\leq 23.3 \mathrm{mag} / \operatorname{arc~sec}^{2}$ \\
\hline Exozodi dust brightness ${ }^{c}$ & 4.5 zodi \\
\hline $\begin{array}{l}{ }^{\mathrm{a}} \text { For the assumed isotropic scatterin } \\
0.3 \text { spherical albedo. } \\
{ }^{\mathrm{b}} \text { The zodiacal light varies with positi } \\
\text { to the minimum at the ecliptic pole } \\
{ }^{\mathrm{C}} \text { The unit of } 1 \text { zodi is equivalent to }\end{array}$ & $\begin{array}{l}\mathrm{c} \text { albedo is equivalent to } \\
\text { yiven value corresponds } \\
c^{2}\end{array}$ \\
\hline
\end{tabular}

The orbital semi-major axes are sampled over a range from inside the inner habitable zone (defined as $0.95 \sqrt{L_{\star}} \mathrm{AU}$, where $L_{\star}$ is the stellar luminosity relative to the Sun) to outside the outer habitable zone (defined as $1.67 \sqrt{L_{\star}} \mathrm{AU}$; Ref. 12). These orbital radii correspond to planet equilibrium temperatures from 261 to $197 \mathrm{~K}$, for an assumed geometric albedo of 0.2 .

The range of planet radii considered is bounded above at $r_{p l} \leq 1.4 r_{\oplus}$, based on evidence suggesting that planets with radii below this value are predominantly rocky. ${ }^{13}$ The lower bound on terrestrial planet radii depends on the planet's ability to retain an appreciable atmosphere, which in turn depends on its stellar illumination. This results in a dependence on the planet's semi-major axis $a_{\mathrm{p}}$, modified by the stellar luminosity, to give ${ }^{14} r_{p l} / r_{\oplus} \geq 0.8 a_{p}^{-1 / 2} L_{\star}^{1 / 4}$.

We model Earth-like exoplanets assuming they scatter light isotropically using a Lambertian illumination phase function with a geometric albedo of 0.2 (Ref. 15). We model the star as a blackbody radiator with the parameters provided in ExoCat. ${ }^{16}$ We include obscuring dust, both in the target system (exozodiacal dust with a fiducial value of 4.5 zodi $^{17}$ ) and locally in the Solar System (zodiacal dust using the model of Ref. 18). While some systems may have exozodiacal dust above this nominal level (e.g., Fomalhaut), the mission's observing plan includes a decision tree to efficiently remove systems from the target list, should their observed dust level preclude Earth-like planet imaging. ${ }^{2}$ Assumed planet and dust characteristics are summarized in Table 1.

While the use of an occulting starshade does allow for detection of fainter and closer planets, it also puts constraints on the allowed times of observation. The telescope-starshade system has a region of allowed Sun angles over which it can operate with the lower limit defined by the exclusion angle from the baffle of the telescope and the outer limit defined by reflection and scattering of sunlight off the starshade into the telescope baffle (Table 2).

\subsection{Observatory}

The integration times are based on the Starshade/ Roman system parameters provided in Table 2. Roman has a telescope diameter of -m resulting in a point spread function of 65 mas at 750-nm wavelength. We assume observations in the 615- to 800-nm band with an end-to-end efficiency, including optical throughput and detector efficiency, of $3.5 \%$ in imaging mode. The Starshade has an IWA of 100 mas, below which planets are assumed to not be observable. This is treated as a sharp cutoff, although in reality there is some throughput below the IWA. ${ }^{19}$ The instrument contrast at the IWA and above is assumed to be $4 \times 10^{-11}$, as calculated by Ref. 1 . Integration times vary between 1 and 6.3 days, depending on the target (explained below).

In addition to the sensitivity, determined by the parameters given above, the main constraints to orbit reconstruction are the nominal mission lifetime of 2 years and the solar exclusion angles. The minimum solar exclusion angle of $54 \mathrm{deg}$ is determined by the telescope baffle while the maximum solar exclusion angle of $83 \mathrm{deg}$ is determined by scattering off the edge of 
Table 2 Mission parameters.

\begin{tabular}{lc}
\hline \hline Parameter & Assumed performance \\
\hline Mission lifetime & 2 years \\
Telescope primary mirror & $2.4 \mathrm{~m}$ \\
Imaging resolution & 65 mas at $750 \mathrm{~nm}$ \\
Imaging bandpass & 615 to $800 \mathrm{~nm}$ \\
Imaging end-to-end efficiency & 0.035 \\
Solar exclusion angle (min) & 54 deg \\
Solar exclusion angle (max) & 83 deg \\
IWA & 100 mas \\
Instrument contrast & $4 \times 10^{-11}$ \\
Imaging integration time & 1 to 6 days \\
\hline \hline
\end{tabular}

the Starshade. For Roman's orbit around the L2 Sun-Earth Lagrange point, the calculated observing windows are shown in Fig. 1. During the 2-year lifetime of the mission, there are generally four opportunities to observe each target. It is assumed that targets with Earth-like planet candidates, following the decision tree laid out in Ref. 2, will be visited once in each of the 4 observing windows available. While spectral characterization can be performed with only a single visit with favorable illumination phase, multiple epochs are needed to constrain the planet's orbit, in particular its semi-major axis, which determines whether the planet is in the habitable zone. To best trace the orbit, the timing of the observations should be as evenly spaced as possible over the orbital period, given the limited observing windows and mission lifetime. The dots in the observing windows in Fig. 1 show the observations times assumed in this study. The retargeting strategy in Ref. 2 assumed that observations can be made within a number of days of the marked dots that would allow for a spectral measurement, which varies from target to target depending on the expected Earth-like exoplanet brightness and observing window length (typically 10 days). It is worth noting that not all observations are required as targets are re-prioritized based on observations. See Ref. 2 for more details.

The properties of the target stars are shown in Table 3. In Ref. 2, we defined the single-visit completeness as the probability that an Earth-like planet would be detected during one target

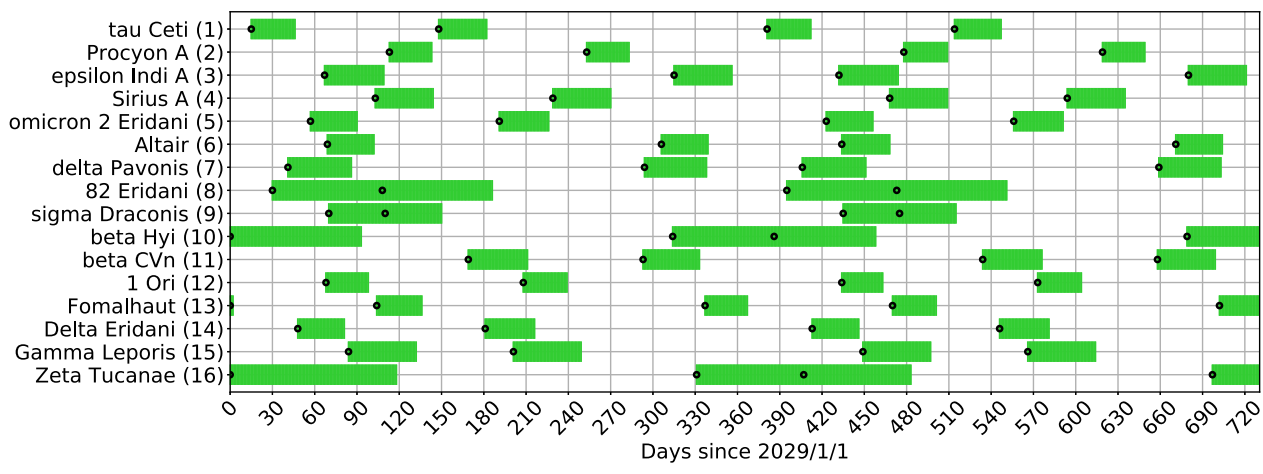

Fig. 1 Target star observing windows (reproduced from Ref. 2) resulting from the telescope and starshade solar exclusion angles. Each target typically has two 30-day-long observing windows per year. Targets at high ecliptic latitude can have longer observing windows per year. The black dots mark the four desired observation start times in a two-year period. This is driven by the need to allow for sufficient time to spectrally characterize a planet if it is bright enough. 


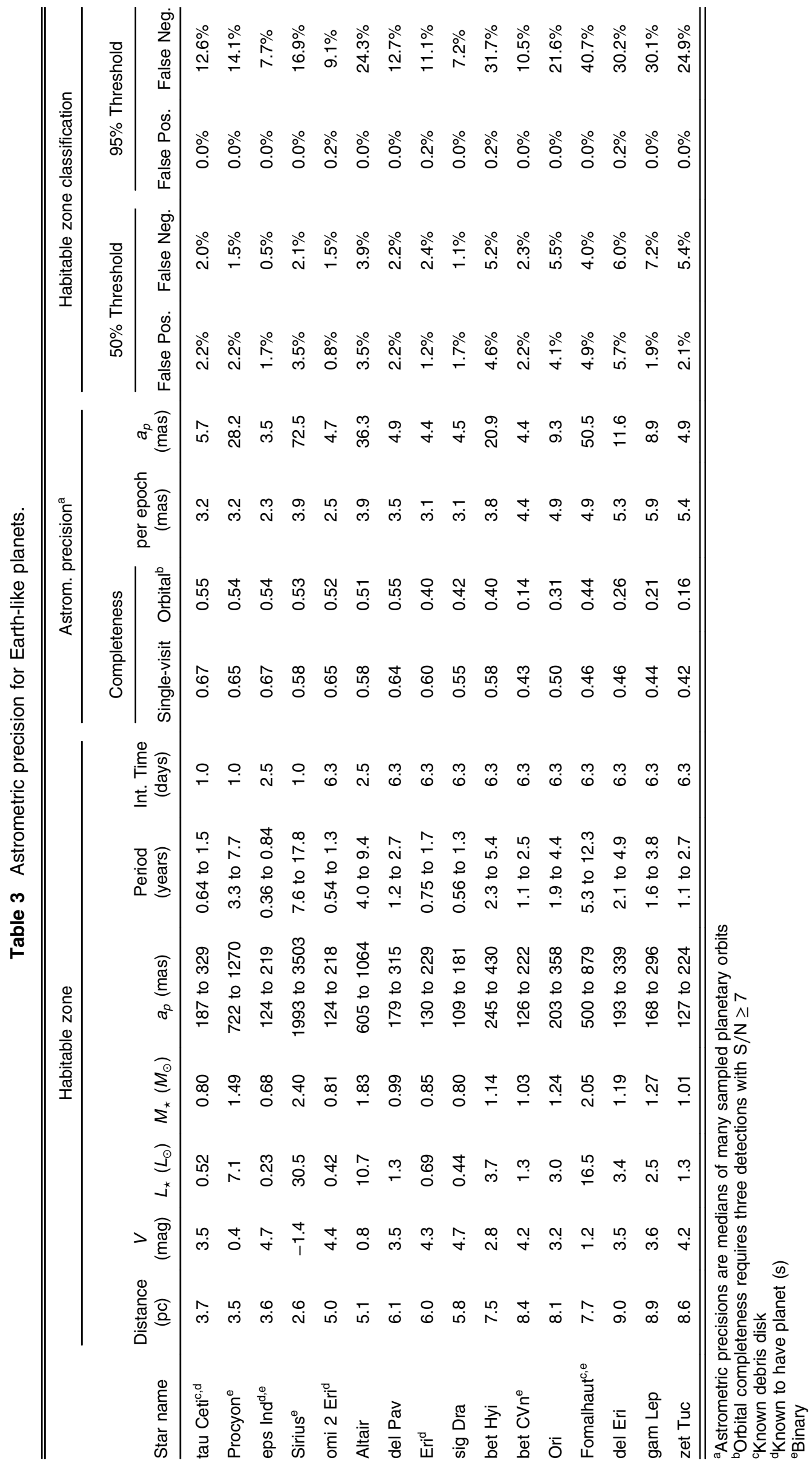


observation at a random time. The imaging integration time for each target is set by identifying what is required to reach a single-visit completeness of at least $50 \%$ with a minimum value of 1 day and a maximum value of 6.3 days (see Table 3 ). We also defined the orbital completeness as the probability that a randomly selected orbit will be detectable $(\mathrm{S} / \mathrm{N} \geq 7)$ during at least 3 of its 4 observing epochs. For most of the targets about half of the planet orbits meet this criterion, but the orbital completeness can sometimes fall below $20 \%$ for systems where the planet signal is relatively weak (again, see Table 3). While the orbital completeness is limited by the orbit geometry (sometimes a planet is only illuminated as a crescent phase and sometimes its projected location is too close to its parent star), it is possible to increase the completeness somewhat with longer integration times for imaging. However, this would limit the time available for characterizing the planet's spectrum, particularly when the observing window is only $~ 30$ days long. With the nominal integration times, about half of the detected Earth-like planets will have sufficient SNR spectra to characterize their atmospheres. ${ }^{2}$

In the next section, we calculate whether at least three detections in four visits (our definition of orbital completeness) is sufficient to determine whether a planet lies within its habitable zone.

\section{Orbit Reconstruction}

Having identified the best targets for detection of Earth-like planets with their observation availability windows, we now describe our approach to orbit reconstruction. We assume the planet is observed at the beginning of each window as shown in Fig. 1, which provides four observing epochs per target in most cases. In cases where the star has a single long availability window per year, we have set the observing times to the beginning and middle of that window.

For each Monte Carlo sampled planet, we propagate its circular orbit to each of the observing epochs and calculate its illumination phase (see Sec. 2). We apply the observatory model to estimate the planet SNR. Observations with SNR $\geq 7$ are considered to be detections. Otherwise, the observation is rejected as a non-detection. The one-dimensional astrometric uncertainty is approximated according to Ref. $20 \delta \theta=(65 \mathrm{mas}) / S N R$. The median astrometric precision for each star ranges from 2.3 to 5.9 mas (see Table 3 ). The simulated data are created by taking the true position of the planet and adding two-dimensional Gaussian scatter based on the astrometric precision, $\delta \theta$.

For the orbit reconstruction, we implemented a forward modeling of Kepler's laws, as described in Ref. 21, into our own software package. We sample all six Keplerian parameters, also including uncertainties in the star's mass and distance, treating them as nuisance parameters. Table 4 lists the parameters that we fit for each orbit, along with their assumed ranges and

Table 4 Orbit fitting parameters.

\begin{tabular}{llcc}
\hline \hline Parameter & \multicolumn{1}{c}{ Description } & Bounds & Prior constraint \\
\hline$a$ & Semi-major axis & 0 to $10 \mathrm{AU}$ & Uniform \\
$e$ & Eccentricity & 0 to 1 & Uniform \\
$\omega$ & Argument of periastron & 0 deg to $360 \mathrm{deg}$ & Uniform \\
$i$ & Inclination $^{\text {a }}$ & 0 deg to $90 \mathrm{deg}$ & $\propto \sin (i)$ \\
$\Omega$ & Longitude of the ascending node & 0 deg to $360 \mathrm{deg}$ & Uniform \\
$T_{0}$ & Periastron phase & 0 deg to $360 \mathrm{deg}$ & Uniform \\
$M_{\star}$ & Stellar mass & 0 to $5 M_{\odot}$ & Observed value with $10 \%$ uncertainty \\
$d_{\star}$ & Distance & 0 to $20 \mathrm{pc}$ & Observed value with $1 \%$ uncertainty \\
\hline \hline
\end{tabular}

$\mathrm{a}_{i}=90$ deg corresponds to edge-on. 

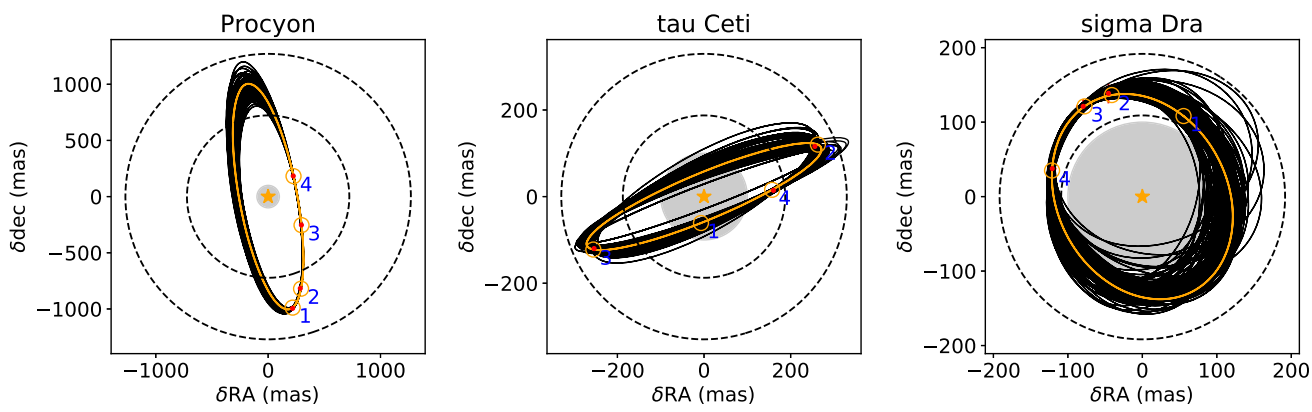

Fig. 2 Example fits are shown for three different stars-Procyon, tau Ceti, and sigma Dra. In each case, the planet's semi-major axis lies within the habitable zone (dashed lines). The true orbit is shown in orange, with true positions marked as orange circles and simulated observations shown with error bars shown in red only if detected. The numbers indicate the visit number for each of the four observations. Sample best-fit orbits are shown as thin black lines. The region masked by the starshade is shown as a gray circle.

prior constraints. We use the emcee Markov chain Monte Carlo (MCMC) software package ${ }^{22}$ to fit the orbit. The MCMC fitting procedure calculates the quality of fit for a series of parameter values, not only converging toward the best set of values but also finding the full parameter ranges that are consistent with the data. Periodic orbital elements $\left(\omega, \Omega, T_{0}\right.$, and $\left.i\right)$ are modulated to stay within their prescribed bounds.

Three examples of the orbit reconstruction simulations are shown in Fig. 2. The first panel shows Procyon, a relatively luminous star $\left(7.1 L_{\odot}\right)$, meaning that its habitable zone is relatively distant both in angular scale $\left(0.7^{\prime \prime}-1.3^{\prime \prime}\right)$ and in physical space (2.5 to $\left.4.5 \mathrm{AU}\right)$. With a mass of $\sim 1.5 M_{\odot}$, planets in the habitable zone have relatively long periods. The randomly selected planet orbiting Procyon is detected in all four observations, but because of the long period, only a fraction of the orbit is traced. The second panel shows a planet orbiting tau Ceti that is only detected in three of the four observing epochs; during the first observation, the planet falls behind the starshade mask (the gray circle in the center of each panel), a common occurrence for planets on inclined orbits. In the third panel (sigma Dra), there are again only three successful observations, but in this case the planet is too faint to be detected during the first epoch due to unfavorable illumination phase. Also, because sigma Dra is near the ecliptic north pole, it has only one observing window per year, although of significantly longer duration (see Fig. 1), and its orbital phase coverage is limited (epoch pairs $1 / 2$ and $3 / 4$ are within the same window). With only three closely spaced epochs, the fit is relatively poorly constrained.

An example of the MCMC posterior distributions is shown in Fig. 3. For this inclined orbit, the inclination $(i)$ and longitude of ascending node $(\Omega)$ are well determined and are accurately retrieved. The retrieved eccentricity $(e)$ is necessarily larger than the assumed circular orbit, but is still close to zero $(0.03 \pm 0.02)$. Given the circular orbit, the true argument of periastron $(\omega)$ is undefined and the retrieved value is only loosely constrained. Most importantly, the semi-major axis $(a)$ is well constrained by the observations, enabling us to determine that the planet lies well within the habitable zone.

\section{Results}

For each of the 16 target stars listed in Table 3, we simulate 1000 random orbits and then extract orbital parameters as described above. Table 5 lists the number of orbital calculations for the full set of simulations (see Ref. 22 for details on MCMC parameters).

Our first objective is to accurately determine each planet's semi-major axis. The ability to make this measurement depends not only on the astrometric precision for individual observations (2.8 to 5.9 mas; Table 3 ), but also depends critically on the orbital sampling. If planets do not trace out their full orbit during the two-year observing window, the quality of the fit is reduced. This is particularly true for stars with high luminosity, which translates to a more distant 

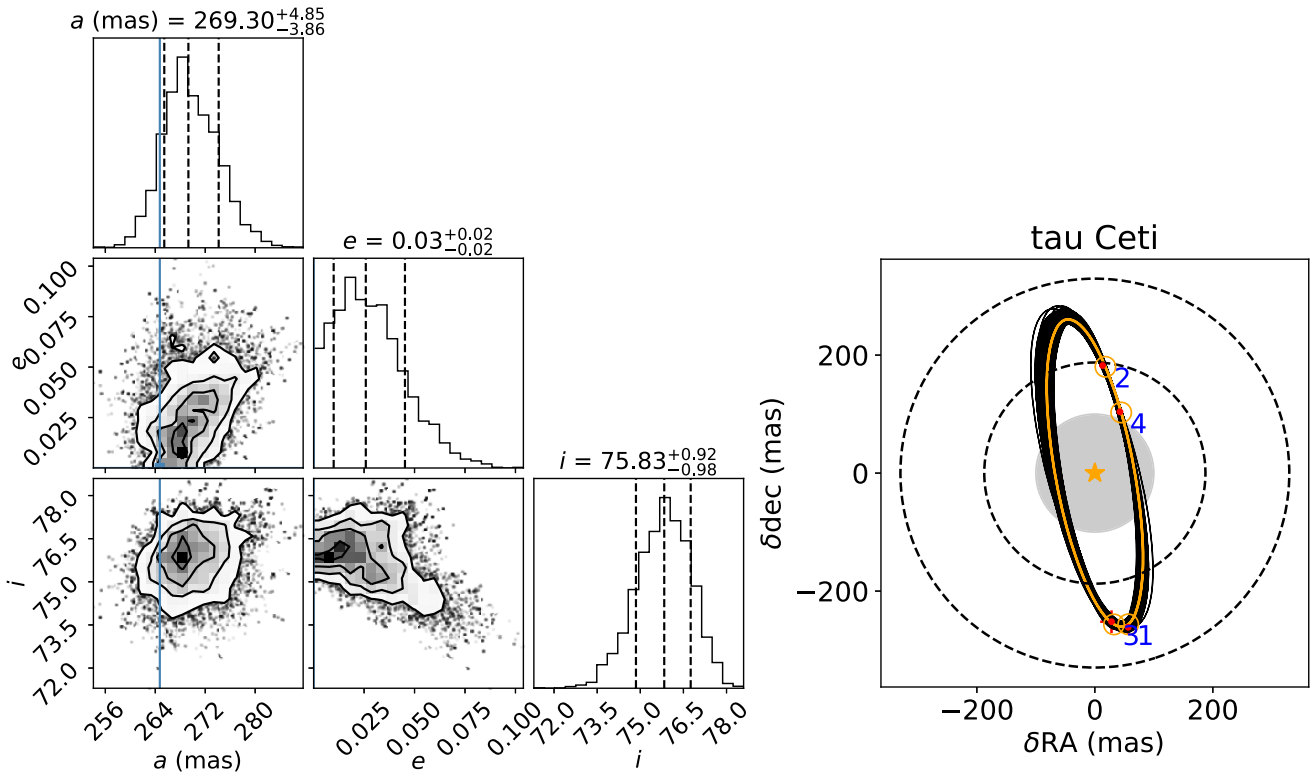

Fig. 3 Best-fit parameters for a random planet orbiting tau Ceti, showing the marginalized probability distributions for 3 of the 8 model parameters (top histograms) and the correlations between pairs of parameters (central panels, with 1-, 2-, and $3-\sigma$ contours). The retrieved parameters are consistent with the true values (blue lines), although the fit eccentricity is necessarily larger than the true circular orbit's. For this example, the planet lies unambiguously inside the habitable zone; the distribution of semi-major axes falls entirely within tau Ceti's 187 to 329 mas range.

Table 5 Simulation parameters.

\begin{tabular}{lc}
\hline \hline Parameter & Quantity \\
\hline Target stars & 16 \\
Random orbits per star & 1000 \\
MCMC iterations per orbit & 5000 \\
MCMC walkers & 100 \\
\hline \hline
\end{tabular}

habitable zone and hence longer orbital periods. Sirius, the most luminous star in our sample (30.5 $\left.L_{\odot}\right)$, has the worst precision in its orbit fitting $(72.5 \mathrm{mas})$, whereas eps Ind, the least luminous star $\left(0.23 L_{\odot}\right)$, has the best determined orbit ( 3.5 mas). Table 3 lists the median semi-major axis precision obtained for the other target stars.

Our ultimate objective is to determine whether a planet lies within its parent star's habitable zone. The key metric for this determination is not the absolute precision, but rather the fractional precision on a planet's semi-major axis. While the absolute precision varies between Sirius and eps Ind by a factor of 20 , the fractional precision for the two is comparable ( $2.7 \%$ and $2.1 \%$, respectively) since Sirius' habitable zone is a factor of 16 larger than eps Ind's. For the overall sample, the median fractional precision varies from $2.1 \%$ up to $7.6 \%$ for Fomalhaut.

Figure 4 shows semi-major axis measurement precision versus true semi-major axis for each of the 1000 simulated planets around three of our target stars. 82 Eri (left panel) has one of the best precisions (4.4 mas median), although the performance degrades significantly for more distant orbits, where the planets are relatively faint. Fomalhaut (central panel) has one of the worst 

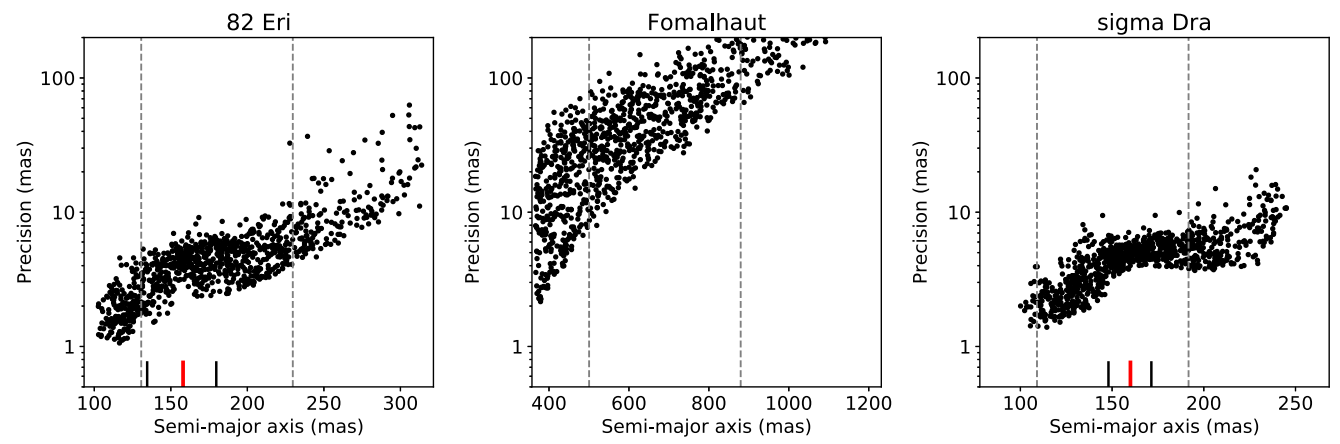

Fig. 4 For each star (three examples shown here-82 Eri, Fomalhaut, and sigma Dra), the orbital parameters and their uncertainties are retrieved for 1000 random planet orbits, each of which is directly imaged at least three times. The precision for measuring the semi-major axis of each planet is shown here as a function of the true semi-major axis. The habitable zone is interior to the dashed lines. The starshade masks all orbits inside of 100 mas. Hash marks at the bottom of each panel correspond to orbital periods equal to the spacing between observing epochs, with a 1-year period highlighted in red.

precisions (50.5 mas median), primarily because the planets in its habitable zone around this A-type star have periods considerably longer than our 2-year mission lifetime (5 to 12 years), such that only a fraction of each orbit is traced. The effect of limited phase coverage can be seen in Figure 5, which plots semi-major axis precision as a function of orbital period in the center of the habitable zone. Planets with periods less than our 2-year mission lifetime are well constrained, but those farther out have semi-major axis precision increasing roughly linearly with the period.

While the semi-major precisions for other target stars (shown in Fig. 8 in the Appendix) follow a similar pattern of smoothly decreasing precision with increasing $a_{p}$, sigma Dra (right panel in Fig. 4) has an unusual bump around $a_{p} \simeq 160$ mas, corresponding to orbital periods of

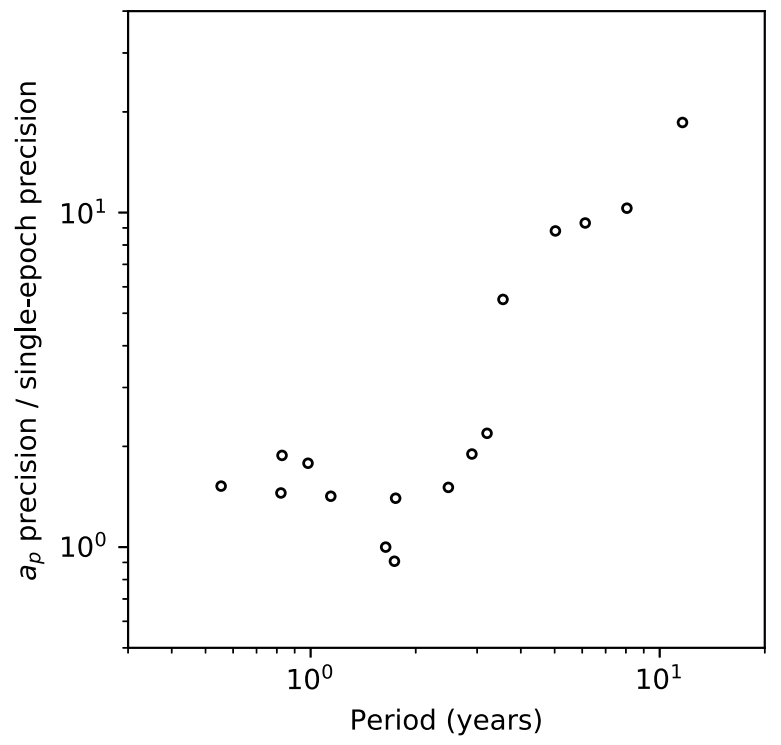

Fig. 5 Our ability to pin down a planet's semi-major axis depends on the fraction of its orbit that is traced by the observations. Periods less than the mission lifetime ( 2 years) are well sampled, whereas those with longer periods are only observed for a partial arc, resulting in lower precision in determining the orbit. 

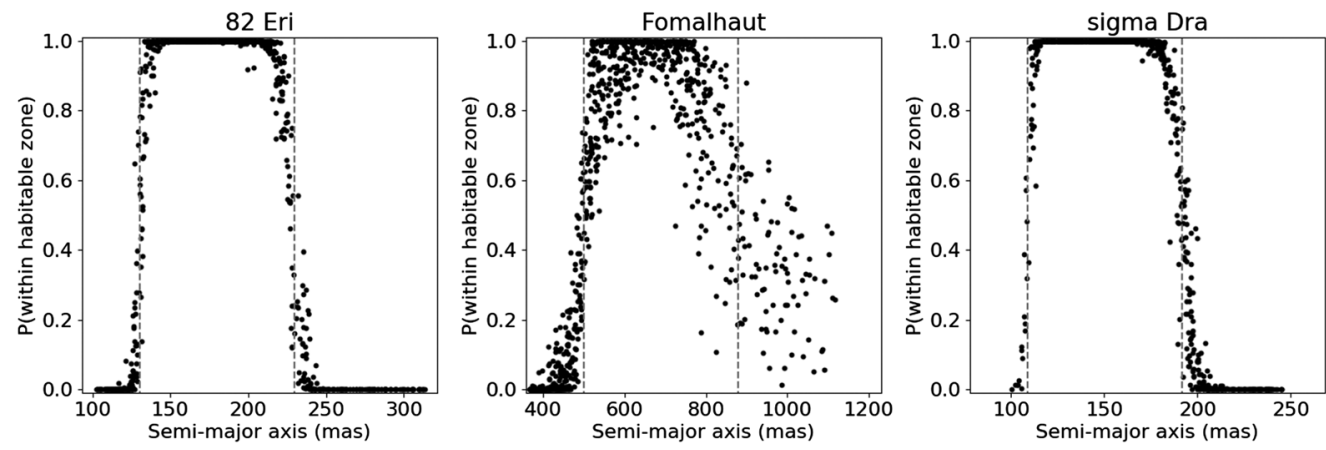

Fig. 6 As in Fig. 4, orbital parameters are measured for 1000 random planetary orbits around each of three stars-82 Eri, Fomalhaut, and sigma Dra. The derived probability of each planet residing in its star's habitable zone is shown as a function of its true semi-major axis. The habitable zone is indicated by the dashed lines.

$\sim 1$ year. The decrease in performance is due to the sampling being repeated on 1-year cycles, where observations taken during the second year of the mission have about the same orbital anomaly as those taken in the first year of the mission (i.e., there is a 1-year aliasing). The resulting small range of orbital-anomaly coverage (see the right panel of Fig. 2) makes it more difficult to fit the orbit.

While the 1-year aliases just discussed is most pronounced for sigma Dra, several other systems exhibit a similar effect at orbital periods that match the phasing of the observations. For all of the precision plots (Figs. 4 and 8), the semi-major axis corresponding to a 1-year period is indicated by a red hash mark; the other black marks correspond to other time differences between observing epochs (e.g., for 82 Eri, the first and second observations are separated by 40 days, the second and third by 325 days, and the first and fourth by 405 days; see the observing windows in Fig. 1). While the 1-year aliasing generally causes the strongest effect, other orbital period/observing period alignments can also degrade performance.

Our MCMC fitting procedure calculates a (non-Gaussian) posterior distribution for each orbital parameter. From these distributions we derive the probability that each planet lies within its star's habitable zone. Figure 6 shows the results for the same three target stars as in Fig. 4. For 82 Eri (left panel), the orbit fitting is fairly deterministic - planets well inside the habitable zone are correctly identified as such with high probability $(>99 \%)$, whereas those well outside are ruled out (probability $<1 \%$ ). As one would expect, there is some ambiguity near the edges of the habitable zone, but for the overall sample there is just a $2.4 \%$ chance of a habitable zone planet being falsely classified as falling outside the habitable zone, whereas $98.8 \%$ of the planets classified as residing in the habitable zone are truly habitable zone planets (i.e., a false positive rate of $1.2 \%)$.

These rates are based on a nominal classification threshold, where planets with habitable zone probability $>50 \%$ are categorized as habitable zone planets. If a more conservative approach is desired, less planets can be included as habitable zone. If a $95 \%$ threshold is used for classification, for example, then 82 Eri will have only $0.2 \%$ false detections. However, only $88.9 \%$ of the true habitable zone planets will be included $(11.1 \%$ false negative rate).

For Fomalhaut (middle panel of Fig. 6), the worse orbital precision translates to much more scatter in the plotted probabilities and less certainty for determining whether a planet lies in its habitable zone. Still, there is only $4 \%$ probability of a habitable zone planet being misclassified, and only a $4.9 \%$ chance of a habitable-zone-classified planet not being truly in the habitable zone. For the conservative (95\%) classification threshold, the false positive rate falls to zero, but at the expense of only $59 \%$ of the true habitable zone planets being included (i.e. a false negative rate of $41 \%$ ).

The false positive and false negative rates for 82 Eri, Fomalhaut, and sigma Dra are shown in Fig. 7, as a function of the classification threshold. These plots also display an overall success 

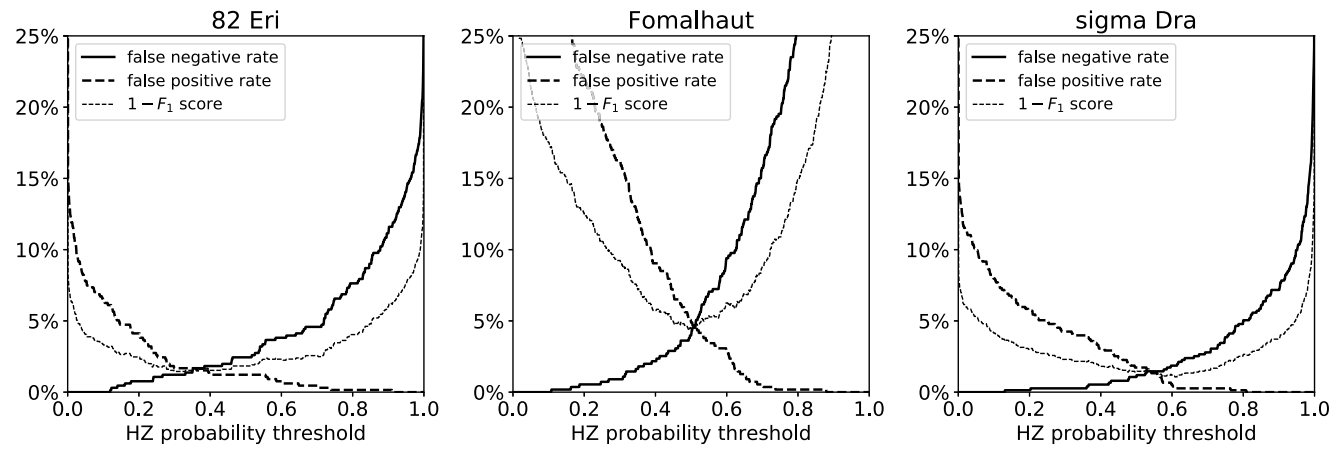

Fig. 7 False positive and false negative rates for 82 Eri, Fomalhaut, and sigma Dra, shown as a function of the habitable-zone probability threshold. A more liberal threshold (lower probability) reduces the false negative rate, but increases the false positive rate. A very conservative threshold (to the right of each panel) can ensure that no false detections are made, but misses a significant number of true habitable-zone planets. A performance metric combining the two rates is given by the $F_{1}$ score, the harmonic mean of the precision (the fraction of detected habitable zone planets that truly are located in the habitable zone) and recall (the fraction of habitable zone planets that are correctly classified as such).

metric - the $F_{1}$ score, the harmonic mean of ( 1 - false positive rate) and ( 1 - false negative rate). While a nominal threshold of 50\% results in an optimal balance between these two factors (i.e., it give maximum $F_{1}$ score), an emphasis on avoiding false detections would warrant a more conservative approach.

The false positive/false negative rates for each star are listed in Table 3 for both the nominal classification threshold (50\%) and a conservative classification threshold (95\%). For the nominal threshold, the average performance for the overall sample is a $2.8 \%$ false positive rate and a $3.3 \%$ false negative rate. For the conservative threshold, the average false positive rate is just $0.05 \%$, but the average false negative rate goes up to $19 \%$.

\section{Discussion}

The results of this study should be considered as a lower bound on the sensitivity since it is based on a simple SNR estimation and has not included the difficulties of image processing for handling non-ideal behaviors. These include stray light from the starshade, solar glint, starlight leakage, fluctuating position errors of the starshade, structure, and gradients in the exozodiacal light, and overlapping exoplanet images in complex systems. These issues are best addressed using imaging simulations such as SISTER $^{23}$ and will be the subject of future work. Many of these issues will be addressed with a community-based data challenge. ${ }^{24}$ A number of these nonideal behaviors have been shown in Ref. 25. Diffraction of sources near or below the IWA could introduce additional astrometric position errors and will need to be studied further in future imaging simulation work. While the upcoming data challenge will focus on issues specific to starshade imaging, it will also consider the more generic problem of interference between multiple planets in a single system. The models and expected performance presented in this work and Ref. 2 will serve as a useful metric to compare against the results of the Starshade data challenge and can provide a base model to estimate the magnitude of systematic uncertainties due to these non-ideal behaviors.

\section{Conclusions}

Based on a model for the SRP mission concept with target-specific observing windows and SNR calculations dependent on the planet illumination during each window, we have quantified 
the ability of SRP to identify habitable zone planets. We find that detection of a planet in at least 3 out of the 4 observing epochs will adequately measure the planet's semi-major axis. For a 16 star sample observed with this strategy, we find that habitable zone planets are correctly identified as such $96.7 \%$ of the time, with $2.8 \%$ contamination by false classifications. Including the full range of planet masses, the mission is expected to detect $\sim 10$ planets in the vicinity of the habitable zone, ${ }^{2}$ such that a very small number of planets $(<1)$ are expected to be misclassified.

\section{Appendix A: Additional Figures}

In this appendix we provide figures for astrometric precision and the probability that an exoplanet is correctly identified as being a habitable zone exoplanet as a function of semi-major axis for all targets considered in this study (Figs. 8 and 9).
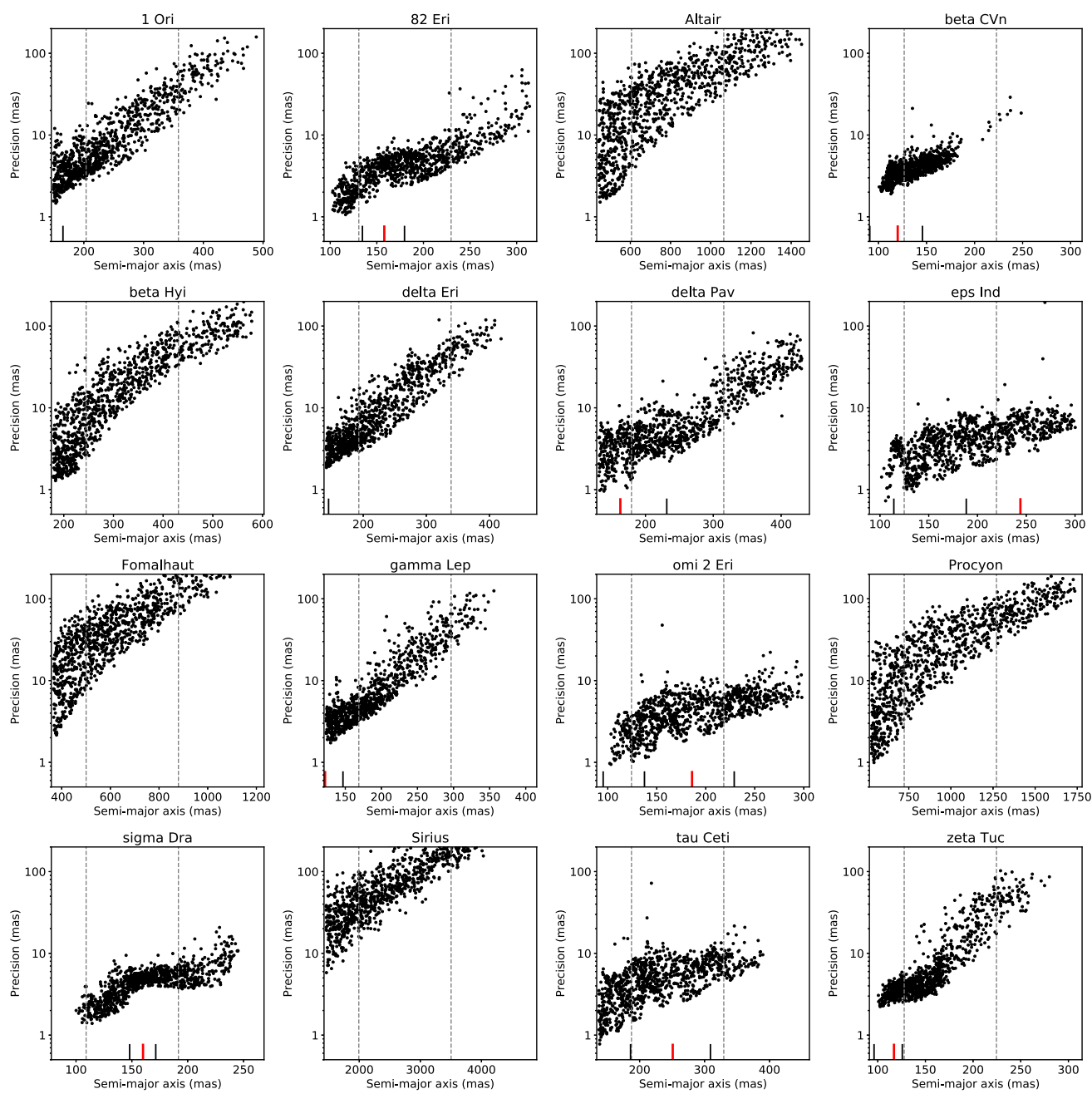

Fig. 8 For each of our 16 target stars, the orbital parameters are retrieved for 1000 random planet orbits, each of which is directly imaged at least three times. The precision for measuring each planet's semi-major axis is shown here. 

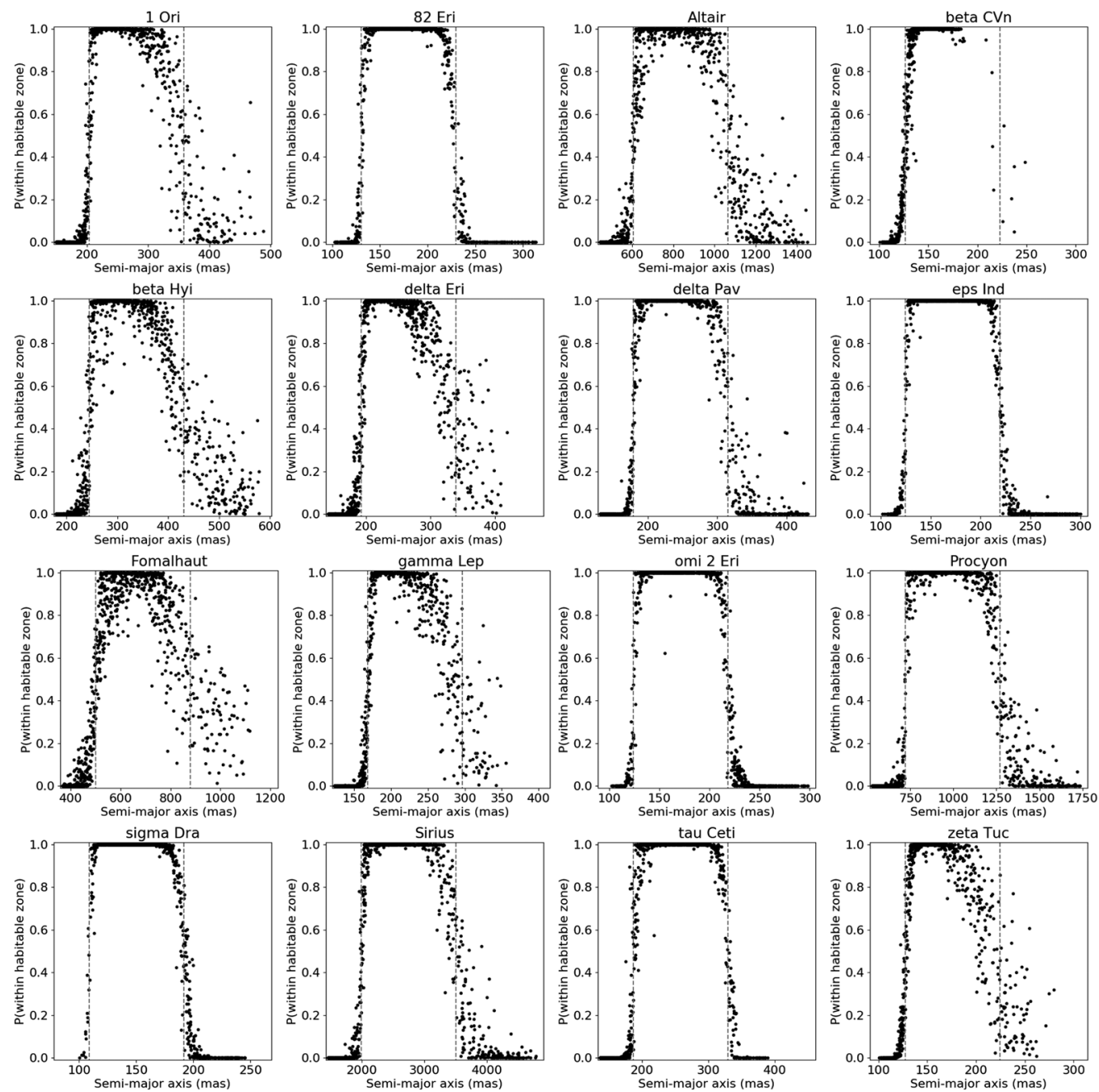

Fig. 9 For each target star, orbital parameters and their uncertainties are measured for 1000 random planetary orbits. The probability of each planet residing in its star's habitable zone is shown as a function of its true semi-major axis.

\section{Acknowledgments}

We thank the reviewers for many insightful comments that have improved the quality of this paper. Part of this work was carried out at the Jet Propulsion Laboratory, California Institute of Technology, under a contract with the National Aeronautics and Space Administration. (C) 2021. All rights reserved. This research has made use of (1) the NASA Exoplanet Archive, which is operated by the California Institute of Technology, under contract with the National Aeronautics and Space Administration under the Exoplanet Exploration Program and (2) the SIMBAD database, operated at CDS, Strasbourg, France.

\section{References}

1. S. Seager et al., "Starshade Rendezvous probe" (2019), https://smd-prod.s3.amazonaws .com/science-red/s3fs-public/atoms/files/Starshade2.pdf.

2. A. Romero-Wolf et al., "Starshade Rendezvous: exoplanet sensitivity and observing strategy," J. Astron. Telesc. Instrum. Syst. 7(2), 021210 (2021).

3. D. Mawet et al., "Deep exploration of $\in$ Eridani with Keck Ms-band vortex coronagraphy and radial velocities: mass and orbital parameters of the giant exoplanet," Astron. J. 157, 33 (2019). 
4. E. B. Ford, "The effects of multiple companions on the efficiency of space interferometry mission planet searches," Publ. Astron. Soc. Pac. 118, 364-384 (2006).

5. O. Guyon et al., "Simultaneous Exoplanet characterization and deep wide-field imaging with a diffractive pupil telescope," Astrophys. J. 767, 11 (2013).

6. S. Blunt et al., "Orbits for the impatient: a Bayesian rejection-sampling method for quickly fitting the orbits of long-period exoplanets," Astron. J. 153, 229 (2017).

7. A. Horning, R. Morgan, and E. Nielson, "Minimum number of observations for exoplanet orbit determination," Proc. SPIE 11117, 111171C (2019).

8. C. M. Guimond and N. B. Cowan, "Three direct imaging epochs could constrain the orbit of Earth 2.0 inside the Habitable Zone," Astron. J. 157, 188 (2019).

9. R. Belikov et al., 2017, https://exoplanets.nasa.gov/exep/exopag/sag/\#sag13.

10. B. S. Gaudi et al., "The Habitable Exoplanet Observatory (HabEx) mission concept study final report," arXiv:2001.06683 (2020).

11. C. C. Stark et al., "Maximized exoEarth candidate yields for starshades," J. Astron. Telesc. Instrum. Syst. 2, 041204 (2016).

12. J. F. Kasting, D. P. Whitmire, and R. T. Reynolds, "Habitable zones around main sequence stars," Icarus 101, 108-128 (1993).

13. L. A. Rogers, "Most 1.6 earth-radius planets are not rocky," Astrophys. J. 801, 41 (2015).

14. K. J. Zahnle and D. C. Catling, "The Cosmic Shoreline: the evidence that escape determines which planets have atmospheres, and what this may mean for Proxima Centauri B," Astrophys. J. 843, 122 (2017).

15. T. D. Robinson and C. T. Reinhard, "Earth as an exoplanet," arXiv:1804.04138 (2018).

16. M. C. Turnbull et al., "The search for Habitable Worlds. 1. The viability of a Starshade mission," Publ. Astron. Soc. Pac. 124, 418-447 (2012).

17. S. Ertel et al., "The HOSTS survey for exozodiacal dust: observational results from the complete survey," Astron. J. 159, 177 (2020).

18. C. Leinert et al., "The 1997 reference of diffuse night sky brightness," Astron. Astrophys. Suppl. Ser. 127, 1-99 (1998).

19. S. B. Shaklan, L. Marchen, and E. Cady, "Shape accuracy requirements on starshades for large and small apertures," Proc. SPIE 10400, 104001T (2017).

20. J. J. Condon, "Errors in elliptical Gaussian fits," Publ. Astron. Soc. Pac. 109, 166-172 (1997).

21. K. Mede and T. D. Brandt, "The Exoplanet simple orbit fitting toolbox (ExoSOFT): an open-source tool for efficient fitting of astrometric and radial velocity data," Astron. J. 153, 135 (2017).

22. D. Foreman-Mackey et al., "emcee: The MCMC Hammer," Publ. Astron. Soc. Pac. 125, 306-312 (2013).

23. S. H. Rafels et al., "SISTER: Starshade imaging simulation toolkit for exoplanet reconnaissance (conference presentation)," Proc. SPIE 11117, 1111700 (2019).

24. R. Hu et al., "Starshade exoplanet data challenge," J. Astron. Telesc. Instrum. Syst. 7(2), 021216 (2021).

25. E. Peretz et al., "Exoplanet imaging performance envelopes for starshade-based missions," J. Astron. Telesc. Instrum. Syst. 7(2), 021215 (2021).

Andrew Romero-Wolf received his BA degree in mathematics and the BA degree in physics (with honors) from the University of Chicago in 2002, a master's in physical science from the University of Chicago in 2005, and a PhD from the University of Hawaii at Manoa in 2010. $\mathrm{He}$ is currently a member of the technical staff at the Jet Propulsion Laboratory, California Institute of Technology in the Deep Space Tracking group.

Geoffrey Bryden is an astronomer at the Jet Propulsion Laboratory (JPL). His research focuses on the detection and characterization of planetary systems beyond the Solar System, in particular the debris left over after planets form. He received his $\mathrm{PhD}$ in astrophysics from the University of California in 1999.

Greg Agnes received his BS from Rensselaer, his MS from University of MD and his $\mathrm{PhD}$ from Virginia Tech. He currently is a senior mechanical systems engineer in the Advanced Deployable Structures group at JPL, where he architects large precision and deployable space instruments. 
Jonathan W. Arenberg is chief mission architect for Science and Robotic Missions at Northrop Grumman Space Systems. His degrees are in physics and engineering, from the University of California, Los Angeles. Arenberg has contributed to the Chandra X-ray Observatory, Starshade and the James Webb Space Telescope and the Astro 2020 strategic missions. He has authored over 200 conference presentations, papers, a recent book, awarded 15 European and U.S. Patents and is an SPIE fellow.

Samuel Case Bradford earned his PhD at Caltech, and his BS degree from UC Berkeley. His $\mathrm{PhD}$ research focused on developing signal processing techniques to assess the stability of systems with changing structural properties. He is currently the supervisor of the Advanced Deployable Structures Group at NASA's Jet Propulsion Laboratory and is a visitor in Aerospace at Caltech. His recent research has been focused on the design, fabrication, and testing of deployable space structures.

John Debes received his BA degree in physics in 1999 from Johns Hopkins University and his $\mathrm{PhD}$ in astronomy and astrophysics from Pennsylvania State University in 2005. He is an ESA/ AURA astronomer and deputy head of the Science Mission Office at the Space Telescope Science Institute. He has authored or co-authored more than 70 refereed papers and one book chapter. His interests include high-contrast imaging of exoplanets and debris disks, as well as the study of planetary systems that survive postmain sequence evolution.

Matthew Greenhouse has been a civil servant astrophysicist at the Goddard Space Flight Center Observational Cosmology Laboratory since 1996. He is a senior member of the James Webb Space Telescope project science team and leads a NASA Strategic Astrophysics Technology project for development of micro-shutter arrays for space-based multi-object spectroscopy.

Renyu Hu is a scientist at the Jet Propulsion Laboratory (JPL) and his research strives to identify and characterize habitable environments in the Solar System and beyond. He is the Starshade scientist of the NASA Exoplanet Exploration Program, providing science leadership to the S5 Starshade Technology Development Activity and managing the Starshade Science and Industry Partnership program. He received his PhD in planetary science from Massachusetts Institute of Technology in 2013.

Jason Rhodes is a senior research scientist at NASA's JPL. He specializes in the development of space missions for cosmology and exoplanets.

John Ziemer received his BSE degree in aerospace engineering from the University of Michigan and his MA and $\mathrm{PhD}$ degrees in mechanical and aerospace engineering from Princeton University with a focus on electric propulsion. He has been at JPL for more than 20 years and is currently a principal systems engineer working on a variety of different concept and technology development efforts.

Biographies of the other authors are not available. 